\title{
Von der Bewirtschaftung der Aufmerksamkeit zur simulativen Demokratie?
}

\section{Politische Visionen - Von Platon zum Global Village}

„Wie wirklich ist die Wirklichkeit?“ (Watzlawick 1976) Mit dieser zum Klassiker gewordenen Frage hat der Philosoph und Psychotherapeut Paul Watzlawick auf amüsante Weise schon vor Jahrzehnten darüber aufzuklären versucht, was Wirklichkeit ist, dass es Wirklichkeit an sich nicht gebe und dass sie eigentlich das Ergebnis menschlicher Konstruktion sei: Wirklichkeit als Produkt von Kommunikation! Auf die Spitze getrieben haben dann die Säulenheiligen der Postmoderne im Gefolge von Michel Foucault die Nietzsche'sche Sentenz, wonach es keine Tatsachen, sondern nur Interpretationen gibt.

Den wissenschaftstheoretischen Streit darüber, wie angemessen eine solche radikal konstruktivistische Auffassung von Welt ist, können wir hier bei Seite lassen. Dass jedoch alles das, was wir für die Wirklichkeit halten, in enger Verbindung mit Kommunikation steht, ist kaum zu bestreiten. Das gilt im alltäglichen Leben ebenso wie in der Politik. In der privaten Lebensführung und mehr noch in Politikvermittlung und Politikwahrnehmung reicht die interpersonale Kommunikation für eine adäquate Wirklichkeitserfassung nicht aus. Wir sind also auf Berichterstattung durch Medien elementar angewiesen. In der Luhmann-typischen Zuspitzung: „Was wir über die Gesellschaft, ja über die Welt, in der wir leben, wissen, wissen wir durch die Massenmedien“" (Luhmann 1995: 5)

Stellt man diese These in einen historischen Kontext, so ist unverkennbar, dass man sich zur Wirklichkeitskonstruktion und -wahrnehmung - sei es als Individuum, als Kollektiv, als Institution oder als Organisation - stets der Vermittlungsinstrumente und Medien bedient hat, die in der jeweiligen Epoche zur Verfügung standen. Das gab dann Anlass zur Hoffnung auf die Emanzipation des Menschen. Andererseits bot es immer wieder Gelegenheit zu kulturkritischem Räsonieren. Ein entsprechender Bogen lässt sich von der Antike bis in die Gegenwart hinein spannen. Man denke nur an eines der frühesten Zeugnisse medialer Besorgnis. Es geht auf keinen geringeren als Platon zurück. In seinem Phaidros-Dialog wirft er die Frage auf, ob die mittelbare Aneignung von Welt durch die Erfindung der Schrift nicht zu Sprach- und Erkenntnisverlusten führe. 
Setzte Platon - insbesondere auch mit seinem Höhlengleichnis - einen Anfangspunkt in der Reflexion darüber, dass es eine unmittelbare und eine mittelbare Wirklichkeit gibt, dass sich Wirklichkeit medial verändert und dass das Bild von Wirklichkeit täuschen kann, so endet das medienspezifische Räsonieren aktuell nicht bei den ebenso kritischen wie hoffnungsgeschwängerten Botschaften über die Folgen der modernen Informations- und Kommunikationstechnologie. Erinnert sei nur an den technikgläubigen Medienphilosophen und Kulturwissenschaftler Marshall McLuhan. Schon vor einem halben Jahrhundert hat er den medientechnischen Fortschritt per se für identisch mit dem Vernünftigen und dem Guten gehalten. Fernseher und Computer wurden von ihm zu einer Art Erlösungsreligion verklärt: Die Welt auf dem Weg zum „Global Village“ (McLuhan 1962)!

\section{Kommunikation - der Operationsmodus von Politik}

Wer sich eher nüchtern mit der Funktionsweise politischer Systeme beschäftigt, dürfte sich mit der visionären Vorstellung einer kollektiven Identität im globalen Dorf schwer tun; weniger hingegen mit dem Befund, dass jedes soziale System zur Sicherung seines Bestandes auf Kommunikation angewiesen ist (vgl. Parsons 1991). Für demokratische Systeme gilt dies in besonderer Weise. Denn Demokratien unterscheiden sich bekanntlich von anderen politischen Ordnungen dadurch, dass sie in Folge ihrer Zustimmungsabhängigkeit in einer besonderen Begründungspflicht stehen. Ihre Legitimation erfolgt nicht nur, aber doch ganz wesentlich durch Kommunikation. Ganz abgesehen davon, dass Kommunikation ein untrennbarer Bestandteil des Politischen selbst ist. Sehr klar zum Ausdruck gebracht hat dies Hannah Arendt: „Reden selbst [müsse] als eine Art Handeln“ aufgefasst werden. Deshalb sei in der Politik die ,prinzipielle Scheidung von Reden und Handeln nicht statthaft" (Arendt 1993: 48), so die neoaristotelisch argumentierende Politikphilosophin.

Was in einer modernen Mediengesellschaft inzwischen als soziale Selbstverständlichkeit anmutet, erschwert die Unterscheidung zwischen Politik und Kommunikation und sollte doch vor technokratischen Politikvorstellungen schützen. Denn ,Politik pur' im Sinne von Entscheidungen, die als alternativlos gelten und als bloße Exekution vermeintlicher Sachzwänge gerechtfertigt werden, das mag es in existentiellen Notlagen geben. Im Alltag demokratischer Normalverhältnisse kommt dies jedoch einer ,präpolitischen Vorstellung“ (Arendt 1960: 33) gleich. Politik und Kommunikation, das sind zwei Seiten einer für die Erzeugung von „Legitimität“" (Sarcinelli 1998) unverzichtbaren Medaille, nicht erst unter den Bedingungen des modernen Medienbetriebs. Deshalb erweist sich das in der deutschen Politik argumentativ inzwischen viel strapazierte TINA-Prinzip (there is no alter- 
native) als ein der Demokratie nicht angemessenes Legitimationsmuster und als Versuch der Kommunikationsverweigerung.

Viel spricht deshalb dafür, Kommunikation ,nicht als Teilbereich von Politik und auch nicht als eine Dimension“ von Politik, sondern als ihren ,zentrale(n) Operationsmodus" (Marcinkowski 2002: 248) zu verstehen. Das tangiert die normative und politisch-institutionelle Dimension (polity) von Politik ebenso wie die gegenwärtig in Deutschland mehr konsensorientierten als streitigen Verfahren und Prozesse politischer Interessenverfolgung und Entscheidungsfindung (politics) sowie die spezifischen Kommunikationsbedingungen bei der Problemlösung in Politikfeldern (policy). Was wie eine platte Selbstverständlichkeit daherkommt, scheint in Teilen der Politikwissenschaft allerdings nicht hinreichend umgesetzt. Von Ausnahmen (vgl. insb. Korte/Fröhlich 2009; Marschall 2011; Sarcinelli 2011) abgesehen gibt es immer noch auflagenstarke Standardwerke zum politischen System und zum Regieren in Deutschland, die den Zusammenhang von Politik und Kommunikation sowie das besondere Beziehungsverhältnis von politischem System und Mediensystem nicht ausdrücklich würdigen oder gar zu einem durchgehenden Thema machen.

Zwar hat sich die politische Kommunikationsforschung in den vergangenen Jahrzehnten disziplinenübergreifend $\mathrm{zu}$ einem attraktiven und ertragreichen Forschungsfeld entwickeln können. Das hatte dann aber vor allem zwei Folgen: Die Beschäftigung mit Politik, Medien und Kommunikation erfuhr im Zuge wissenschaftlicher Professionalisierung (vgl. als Überblick u. a. Marcinkowski/Pfetsch 2009) eine ,Versozialwissenschaftlichung ' mit zunehmender Fokussierung auf empirisch überprüfbare und nicht selten mikroanalytische Spezifika. Verstärkt wurde diese Entwicklung durch die Abwanderung von zahlreichen Politikwissenschaftlerinnen und Politikwissenschaftlern in die karriereträchtigeren Medien- und Kommunikationswissenschaften.

Nun sind die Grenzen zwischen den sozialwissenschaftlichen Disziplinen fließend. Dennoch verfolgen diese je eigene Fragestellungen mit der Konsequenz, dass spezifisch politikwissenschaftliche Problemstellungen nicht immer im Zentrum des Erkenntnisinteresses stehen. Auch wenn eine über die Zeit angestiegene Kommunikationsabhängigkeit der Politik kaum strittig sein dürfte, so ist doch unverkennbar, dass die Eigenlogik des Politischen, dass politisch-institutionelle und politisch-kulturelle Kontexte nicht immer die notwendige Aufmerksamkeit erfahren, wenn es um die wissenschaftliche Beschäftigung mit politischem Handeln und Entscheiden unter Bedingungen einer modernen Mediengesellschaft geht. 


\section{Medienpräsenz - eine unsichere Machtprämie}

Politische Akteure und Institutionen bemühen sich inzwischen intensiv um ihr eigenes Politikbild und betreiben mit zum Teil erheblichem Aufwand Öffentlichkeitsarbeit und Imagepolitik. Dabei bleibt die Politik, will sie sich öffentlich legitimieren, mangels eigener Medien auf die Berichterstattung durch die Massenmedien angewiesen. Denn trotz der dynamischen Entwicklung auf dem Social MediaMarkt sind es bis auf weiteres ganz überwiegend die allgemein zugänglichen Medien, die Öffentlichkeit herstellen. Es gehört zwar zu den Ritualen der Politikerrhetorik, gegen stimmungsdemokratische Einflüsse immun zu sein. Danach politisches Handeln gänzlich auszurichten, würde die Selbstaufgabe eigener politischer Handlungskompetenz dokumentieren. Mit dem völligen Ignorieren der Ausschläge des Stimmungsbarometers würden sich Akteure aber ins politische Abseits befördern. Insofern gehört die kontinuierliche Beobachtung des Meinungsmarkes, sei es auf Basis der Medienresonanz oder auf Grundlage demoskopischer Erhebungen, zum selbstverständlichen politischen Alltagsgeschäft.

Zur Selbst- und Fremdbeobachtung ist die allgemeine Berichterstattung unverzichtbar. Nicht zuletzt geht es dabei auch und vor allem um die Machtprämie Medienpräsenz. Was medial nicht vorkommt, ist der Kommunikation entzogen. Allerdings erweist sich die Machtprämie Medienpräsenz als eine zunehmend unsichere „Legitimationsressource“ (Martinsen 2009: 38). Dafür sind nicht allein die dynamischen Veränderungen des Mediensystems ausschlaggebend. Auch die damit in Verbindung stehenden gesellschaftlichen, politisch-kulturellen Wandlungsprozesse nähren Legitimitätszweifel bei den Bürgern und erhöhen die „Legitimitätsempfindlichkeit" (Sarcinelli 1998: 263) des demokratischen Systems.

Zentrale Antriebskräfte für den Wandel des Mediensystems sind die weitgehende Liberalisierung in Verbindung mit einer fortschreitenden Kommerzialisierung des Medienmarktes. Die Zahl der Anbieter nimmt zu. Gleiches gilt für die Individualisierung von Nutzungsformen vor allem im elektronischen Bereich. Hinzu kommt eine Angebotsdifferenzierung im Print- und elektronischen Bereich bei gleichzeitiger Konzentration der Besitzerverhältnisse in verschachtelten Medienkonglomeraten. Medienhäuser werden schon lange nicht mehr geprägt von starken Verlegerpersönlichkeiten, die sich über Jahrzehnte hinweg (z. B. Augstein, Bucerius, Springer) als Vorkämpfer einer politisch-weltanschaulichen Grundorientierung verstanden, entsprechend verlegerisch handelten und dabei zur langjährigen Quersubventionierung ihrer publizistischen ,Flaggschiffe“ bereit waren. Medienhäuser verhalten sich heute vor allem marktkompatibel und verstehen sich als Content-Provider. Das gilt inzwischen selbst für den finanziell privilegierten öffentlich-rechtlichen 
Von der Bewirtschaftung der Aufmerksamkeit zur simulativen Demokratie?

Rundfunk. Medienprodukte sollen auf vielen Plattformen angeboten und möglichst käuflich erworben werden können.

Insgesamt ist die Medienlandschaft in Deutschland nicht nur unübersichtlicher und unberechenbarer geworden. Die Entwicklung führt auch dazu, dass sich das Verhältnis zwischen Medien und Politik entkoppelt. Lange gepflegte, symbiotische Tauschgeschäfte mag es noch geben. Die Beziehungen sind aber unberechenbarer geworden. Das zeigt sich etwa in publizistischen Kampagnen auf der Basis einstmals undenkbarer ,Bündnisse', die sich in einer zeitweise erstaunlich politisch konsonanten Berichterstattung und Kommentierung ganz unterschiedlicher Organe (z. B. FAZ, BILD, Spiegel) niederschlagen. Das gilt etwa in Wahlkampfzeiten oder bei spektakulären bzw. zum Spektakulum gemachten Ereignissen, bestimmt aber inzwischen auch das alltägliche Nachrichtengeschäft. Möglich geworden ist dies, weil die Logik des Marktes mehr Distanz zum politischen System schafft. So werden auch lange gepflegte Loyalitäten und symbiotische Beziehungen zu politischen Akteuren und Institutionen aufgegeben, weil - dem ökonomischen Kalkül folgend Publikumsorientierung an Bedeutung gewonnen hat. Bisweilen kann dies dann makabre Züge annehmen, wenn sich der Boulevard (BILD) - wie etwa im Falle des Sturzes des ehemaligen Bundespräsidenten Wulff - als Hüter journalistischer Ethik geriert. Inzwischen räumen selbstkritische Journalisten ein, dass auch die Bereitschaft zur Übertretung professionsspezifischer Spielregeln des Journalismus zugenommen hat, vor allem im journalistischen Getummel der ,Berliner Republik‘.

\section{Berichterstattung - mehr als die Bewirtschaftung von Aufmerksamkeit}

Mehr denn je geht es um die Bewirtschaftung von Aufmerksamkeit. Hier gibt es gewiss noch deutlich unterschiedliche Maßstäbe. Insofern sind Berichterstattung und Kommentierung der Boulevardmedien, der seriösen Tagespresse und vor allem der überregionalen Elitezeitungen nicht, über einen Kamm zu scheren'. Letztere haben trotz allem Rationalisierungs- und Anpassungsdruck an veränderte Rezeptionsgewohnheiten immer noch vergleichsweise hohe Qualitätsansprüche. Und sie repräsentieren mit ihrer Grundausrichtung immer noch einigermaßen das demokratische Spektrum in Deutschland. Auch bei den elektronischen Medien Fernsehen und Hörfunk gibt es erhebliche Unterschiede bei den Politikanteilen in Berichterstattung und Gesamtprogramm. So liegen die Informationsanteile - freilich in sehr großzügiger Verwendung des Informationsbegriffs - in den öffentlich-rechtlichen Medien bei immerhin rund der Hälfte des Programms. Insgesamt aber hat die Distanz zu allem Politischen, sofern es keinen Aufmerksamkeit zu erwartenden Nachrichtenwert verspricht, zugenommen. 
Apropos Nachrichtenwert: Auch die Nachrichtenfaktoren unterliegen einem Wandel. Denn Nachrichtenfaktoren werden nicht allein von unstrittigen, journalistisch-subjektiven oder vermeintlich politischen-objektiven Relevanzkriterien bestimmt. Ebenso wichtig sind Veränderungen in den Rezeptionsgewohnheiten und Publikumserwartungen. Schließlich ist es das Medienpublikum, das durch sein Informationsverhalten und damit letztlich durch Auflagen, Einschaltquoten und Kaufinteresse Rahmenbedingungen für journalistische Angebote setzt. Insofern verdient die zunehmende ,Infizierung' auch der politischen Berichterstattung mit prominenzierenden, boulevardesken und entertainisierenden Elementen nicht nur eine Kritik an Angebot und Anbietern, sondern auch an Nachfrage und Mediennutzern. Mediennutzer aber sind wir alle, wir als Bürger, bekanntermaßen ausgestattet mit einem zwiespältigen Charakter. Insofern kommt es auch darauf an, wie sich der informationsorientierte kritische „Citoyen“ gegen den unterhaltungsorientierten „Bourgeois“ in jedem einzelnen von uns behauptet.

\section{Herstellung von Öffentlichkeit - mehr als empörungsdemokratische Publizität}

Nun ist ein zunehmender Abstand der Medien zur Politik normativ durchaus erwünscht, weil er der Wahrnehmung des politischen Wächteramtes entspricht. Allerdings zeigen sich im Zuge der hier nur angedeuteten Entwicklungen auch deutliche Züge eines Rudel- und Kampagnenjournalismus. So werden dann Tendenzen einer temporär erzeugten Erregungsöffentlichkeit gefördert, die alle Aufmerksamkeit auf ein tatsächliches oder vermeintliches Skandalon lenkt. Die Grundzüge einer durch die Medienberichterstattung begünstigten Empörungsdemokratie hat Richard von Weizsäcker schon vor einem Vierteljahrhundert treffend auf den Begriff gebracht, als er mit Blick auf den seinerzeitigen Medienbetrieb von einem „unheilvollen Umkehrprozess der Wichtigkeiten“ (Weizsäcker 1992: 157) sprach. Die Bewirtschaftung von Aufmerksamkeit mit der Konzentration auf vermeintlich Wichtiges erzeugt dabei gleichzeitig eine „Verunaufmerksamung“ (Mückenberger 2014: 7), also ein Nicht-Wahrnehmen und schnelles Vergessen.

Mehr denn je lohnt es deshalb, an die zentralen Funktionen einer demokratischen Öffentlichkeit zu erinnern, deren Herstellung die eigentliche Aufgabe der Berichterstattung in einem freien Mediensystem sein sollte (vgl. Neidhardt 1994). So ist es zunächst die Beobachtungsfunktion von Öffentlichkeit, die angesichts zunehmender Unübersichtlichkeit die Selbst- und Fremdbeobachtung von Politik und Gesellschaft ermöglicht und im Idealfall ein Forum bietet, das nicht nur den Zugang offen hält für die Akteure des organisierten politischen Betriebs, sondern auch als Resonanzboden für zivilgesellschaftliches Engagement dient. Öffentlichkeit kommt 
Von der Bewirtschaftung der Aufmerksamkeit zur simulativen Demokratie?

zweitens in einem politisch-kulturell veränderten Umfeld eine wichtiger werdende Validierungsfunktion zu. Damit verbindet sich die Erwartung einer durch Berichterstattung gewährleisteten sachlichen Richtigkeit ebenso wie eines gewissen Qualitäts- bzw. Reflexionsniveaus. Drittens sollte die medial hergestellte Öffentlichkeit im Stimmengewirr konkurrierender Aufmerksamkeits- und Zustimmungsangebote eine Orientierungsfunktion bieten. Dabei gilt es, nicht nur die publizistische Plattform für den demokratischen Wettbewerb um politische Positionsgewinne bereitzustellen, sondern auch Informationen und Gründe für Zustimmung bzw. Kritik und Ablehnung.

Beobachtung, Bewertung und Orientierung, das also sind die funktionalen Anforderungen an eine politische Berichterstattung, die mehr ist als empörungsdemokratische Publizität; die der Auseinandersetzung über Angelegenheiten der Allgemeinheit dienen und damit der Beratung und Vorbereitung kollektiv verbindlicher Entscheidung Raum geben. Nur so kann eine Grundlage für den nie abgeschlossenen Prozess der Herstellung von Legitimität im demokratischen System geschaffen werden.

\section{Veränderte Legitimationsarchitektur-Orientierungsbedarf und Transparenz}

Was sich mit Blick auf die Defizite in der Herstellung von Öffentlichkeit durch das Mediensystem als Problem der Legitimationsbeschaffung erweist, korrespondiert mit dem, was üblicherweise als gesellschaftliche Modernisierung bezeichnet wird. Denn die hat gravierende Veränderungen im politischen Verhalten zur Folge. Schon seit geraumer Zeit gehört die politische Auszehrung tradierter politisch-kultureller Milieus zu den Standardbefunden politisch-soziologischer Gesellschaftsanalyse. Konstatiert wird die schleichende, wenn auch keineswegs völlige, Ablösung politischen Verhaltens von Schicht- und Milieubindungen. Politisches Wissen und politische Überzeugungen geraten verstärkt in Abhängigkeit zu eigenen, subjektiven Wahrnehmungen. Von Tradition und soziokulturellen Banden gelöst steht das ,Bild“ von Politik mehr denn je unter dem Einfluss der Medienberichterstattung. Kurz: Die Bürger orientieren sich mehr am Medien- und Meinungsmarkt, weil die Ankerfunktion und die Filterwirkung politisch-weltanschaulicher Grundorientierungen schwächer werden. Dass die öffentliche bzw. veröffentlichte Meinung in einer offenen Gesellschaft, die zu allen Sinn vermittelnden Institutionen auf Distanz geht und vorgegebenen Wahrheitsansprüchen mehr und mehr misstraut, zu einer Art „Wahrheitsäquivalent“ (Luhmann 2000: 280) werden und dabei kollektiven Irrtum erzeugen kann, erscheint dann doch als eine ernstzunehmende Problemanzeige des verstorbenen Ironikers und ,Großmeisters` der Systemtheorie Niklas Luhmann. 
Hingegen mag der Generalverdacht, nach dem Öffentlichkeit geradezu ,ein Symbol für die durch Transparenz erzeugte Intransparenz" sei (Luhmann 2000: 285), in diesem Zusammenhang wie politischer Zynismus anmuten. Dass jedoch mehr Transparenz nicht automatisch demokratische Willensbildung und verantwortliches Entscheiden positiv beeinflusst, sondern auch Konfusion, Konformismus und Hinterzimmerpolitik befördern können, das zeigen die Erfahrungen aus den Anfangszeiten der Partei Die Grünen ebenso wie aktuell das politische Schicksal der PiratenPartei. Letztere ist nicht zuletzt an dem eigenen Anspruch, via „Liquid Democracy“ als Partei ein offenes Kommunikationssystem zu sein, umfassende Partizipation und Transparenz zu realisieren, schon innerparteilich gescheitert - zumindest vorerst.

Totale Transparenz erweist sich in der realen demokratischen Willensbildung als Transparenzillusion, ein regelmäßig entzaubertes und Enttäuschungen produzierendes, vermeintliches Ideal. Denn politische Willensbildung und Regierungshandeln ist immer auch mit Interessenvermittlung und -ausgleich sowie mit der Entwicklung von Entscheidungsalternativen unter Wettbewerbsbedingungen verbunden. Sie steht freilich auch in der ständigen Versuchung, mit arkanpolitischer Attitüde legitime Informations- und Kommunikationsansprüche zu verweigern. Insofern ist das stets neu auszulotende, prinzipiell aber nicht aufzulösende Spannungsverhältnis zwischen „Publizität und Diskretion“ (vgl. Sarcinelli 2011: 75-88) eine funktionale Notwendigkeit des demokratischen Prozesses.

Bedenkenswert ist dieses Spannungsverhältnis noch in einem Zusammenhang, den Michael Zürn mit Blick auf die Perspektiven des Regierens in westlichen Demokratien als „Verschiebung in der Legitimationsarchitektur politischer Herrschaft zuungunsten originär demokratischer Legitimationsquellen" (Zürn 2011: 615) bezeichnet. Sein Befund: Während die „Kerninstitutionen der Mehrheitsbildung“ zunehmend kritisch beäugt würden, hätten ,die Institutionen mit gesamtgesellschaftlicher Entscheidungskompetenz, die dem politischen Prozess entzogen sind, wie etwa Zentralbanken und Verfassungsgerichte, überall ein deutlich höheres Ansehen als die demokratischen Kerninstitutionen“ (ebenda: 613). In Deutschland steht denn auch das Bundesverfassungsgericht genauso wie etwa Zentralbank, Polizei oder Bundeswehr bei den Menschen hoch im Kurs. Dabei erscheint das honorige Image in demokratietheoretischer Hinsicht durchaus ambivalent. Denn ausgerechnet die Institutionen, in denen medienöffentliche Kommunikation zur Kernkompetenz (Parlament, Regierung, Parteien) gehört, genießen nur geringes Ansehen und werden damit - zumindest in der Wahrnehmung der Öffentlichkeit - marginalisiert. Damit ergibt sich die irritierende Erkenntnis, dass Legitimitätszweifel offenbar durch medienöffentliche Kommunikation nicht nur beseitigt, sondern offenbar auch befördert werden können. 
Von der Bewirtschaftung der Aufmerksamkeit zur simulativen Demokratie?

\section{Ein neuer Strukturwandel der Öffentlichkeit-mehr als simulative Demokratie}

Welche Schlussfolgerungen lassen sich aus dieser Skizze zum Verhältnis von medialer Wirklichkeitskonstruktion und Politik ziehen? Mit seiner Metapher vom „Global Village“ (McLuhan 1962) hat der eingangs erwähnte Medientheoretiker Marshall McLuhan schon früh und mit visionärer Kraft auf eine neue Qualität von medialer Wirklichkeitsvermittlung in einer weltweit elektronisch vernetzten Kommunikation hingewiesen. Und er prognostizierte, dass sich damit irgendwann einmal kollektive Identität einstelle oder herstellen lasse. Als eine von Idealismus geprägte philosophische Idee mag das nicht ohne Reize sein. Aus sozialwissenschaftlicher Sicht erscheint diesbezüglich mehr Skepsis als Zuversicht begründet. Nicht strittig dürfte hingegen sein, dass es in Zeiten global vernetzter Kommunikation „keine Unschuld des Nicht-Wissens“ (Emcke 2014) als Rechtfertigung mehr geben kann. Kennzeichen der Zeit sind Schnelligkeit und Allgegenwart im Informationsaustausch. Das betrifft den privaten Informationsaustausch ebenso wie den professionellen Journalismus, der mehr und mehr unter dem Druck von Echtzeitkommunikation steht.

Die Herstellung von Öffentlichkeit muss mehr sein als die Bewirtschaftung von Aufmerksamkeit und mehr auch als die Simulation von Demokratie. Eine zum Echtzeitjournalismus umgepolte, Distanz vermissende und Reflexion verhindernde Berichterstattung taugt ebenso wenig wie ein durchkommerzialisierter Medienmarkt als Basis für Deliberation und Partizipation. Die dynamischen Veränderungen vor allem auf dem Markt der elektronischen Informations- und Kommunikationsmedien, für die es - zumindest technologisch - keine Kapazitätsgrenzen zu geben scheint, fordern den politischen Journalismus ebenso wie die Politik selbst heraus. Sie stellen aber weder die Grundregeln guter journalistischer Arbeit noch die Prinzipien für demokratische Politik in Frage; zumindest sollten sie es nicht können.

Weil der Orientierungsbedarf der Bürger und der Diskursbedarf für eine demokratische Öffentlichkeit, die diesen Namen verdient, steigt, erscheint ein neuer „Strukturwandel der Öffentlichkeit“ geboten, um einen Klassikertitel von Jürgen Habermas zu bemühen. Dann aber muss politische Publizistik mehr bieten als ein Aufmerksamkeit absorbierendes Ereignis-Hopping. Mit ein wenig mehr Sensibilität für den Zeitbedarf von Deliberation, Verfahren und Entscheidungen demokratischer Politik wäre schon viel gewonnen. Wenn politische Berichterstattung aber weiterhin zur bloßen Bewirtschaftung von Aufmerksamkeit verkommt, dann wird sie zur moralischen Zumutung, weil sie allenfalls noch das unbeteiligt-Beteiligtsein fördert. Das aber wäre ein Zustand, der dann allenfalls noch als "simulative Demokratie“ (vgl. Blühdorn 2013) bezeichnet werden kann. 


\section{Literatur}

Arendt, Hannah, 1960: Vita activa oder vom tätigen Leben, Stuttgart.

Arendt, Hannah, 1993: Was ist Politik? Aus dem Nachlaß herausgegeben von Ursula Ludz, München.

Blühdorn, Ingolf, 2013: Simulative Demokratie. Neue Politik nach der postdemokratischen Wende, Berlin.

Emcke, Carolin, 2014: Hinschauen, Süddeutsche Zeitung, 18./19. Oktober 2014, 5. Korte, Karl-Rudolf/Fröhlich, Manuel, 2009: Politik und Regieren in Deutschland, Paderborn u. a.

Luhmann, Niklas, 1995: Die Realität der Massenmedien, Opladen.

Luhmann, Niklas, 2000: Die Politik der Gesellschaft, Frankfurt a. M.

Marcinkowski, Frank, 2002: Politische Kommunikation und politische Öffentlichkeit. Überlegungen zur Systematik einer politikwissenschaftlichen Kommunikationsforschung, in: ders. (Hrsg.), Politik der Massenmedien. Heribert Schatz zum 65. Geburtstag, Köln, 237-256.

Marcinkowski, Frank/Pfetsch, Barbara (Hrsg.), 2009: Politik in der Mediendemokratie. PVS-Sonderheft 42, Wiesbaden.

Marschall, Stefan, 2011: Das politische System Deutschlands, Stuttgart.

Martinsen, Renate, 2009: Öffentlichkeit in der „Mediendemokratie“, in: Frank Marcinkowski/Barbara Pfetsch (Hrsg.), Politik in der Mediendemokratie. PVSSonderheft 42, Wiesbaden, 37-69.

McLuhan, Marshall, 1962: The Gutenberg Galaxy. The Making of Typographic Man, London.

Mückenberger, Ulrich, 2014: Zeiten der Politik und Zeiten der Medien, in: Aus Politik und Zeitgeschichte, B 22-23/2014, 3-9.

Neidhardt, Friedhelm, 1994: Öffentlichkeit, öffentliche Meinung, soziale Bewegungen, in: Kölner Zeitschrift für Soziologie und Sozialpsychologie, Sonderheft 34/1994, Opladen, 7-41.

Sarcinelli, Ulrich, 1998: Legitimität, in: Otfried Jarren/Ulrich Sarcinelli/Ulrich Saxer (Hrsg.), Politische Kommunikation in der demokratischen Gesellschaft. Ein Handbuch mit Lexikonteil, Opladen/Wiesbaden, 254-267.

Sarcinelli, Ulrich, 2011: Politische Kommunikation in Deutschland. Medien und Politikvermittlung im demokratischen System, Wiesbaden (3., erw. und überarb. Aufl.).

Watzlawick, Paul, 1976: Wie wirklich ist die Wirklichkeit - Wahn, Täuschung, Verstehen, München. 
Weizsäcker, Richard von, 1992: Im Gespräch mit Gunter Hofmann und Werner A. Perger, Frankfurt.

Zürn, Michael, 2011: Perspektiven demokratischen Regierens und die Rolle der Politikwissenschaft im 21. Jahrhundert, in: PVS 52 (4), 603-635.

Korrespondenzanschrift:

Prof. em. Dr. Ulrich Sarcinelli

Universität Koblenz • Landau, Campus Landau

Institut für Sozialwissenschaften

Abt. Politikwissenschaft

Kaufhausgasse 9

76829 Landau

E-Mail: sarci@uni-landau.de 